\title{
Chip Error Pattern Analysis in IEEE 802.15.4
}

\author{
Kaishun $\mathrm{Wu}^{* \dagger}$, Haoyu Tan*, Hoi-Lun Ngan* and Lionel M. Ni* \\ ${ }^{*}$ Department of Computer Science and Engineering, \\ Hong Kong University of Science and Technology \\ Email: \{kwinson,hytan,cpeglun,ni\}@ cse.ust.hk \\ ${ }^{\dagger}$ School of Physics and Engineering, \\ Sun Yat-sen University, Guangzhou, China
}

\begin{abstract}
IEEE 802.15.4 standard specifies physical layer (PHY) and medium access control (MAC) sublayer protocols for low-rate and low-power communication applications. In this protocol, every 4-bit symbol is encoded into a sequence of 32 chips that are actually transmitted over the air. The 32 chips as a whole is also called a pseudo-noise code (PN-Code). Due to complex channel conditions such as attenuation and interference, the transmitted PN-Code will often be received with some PNCode chips corrupted. In this paper, we conduct a systematic analysis on these errors occurring at chip-level. We find that there are notable error patterns corresponding to different cases. Recognizing these patterns will enable us to identify the channel condition in great details. We believe that understanding what happened to the transmission in our setup can potentially bring benefit to channel coding, routing and error correction protocol design.
\end{abstract}

Index Terms-IEEE 802.15.4; physical layer (PHY); pseudonoise (PN) codes; chip error patterns; measurement study

\section{INTRODUCTION}

Unlike the wired counterpart, a wireless link is easily affected by environment changes or surrounding wireless activities. Determining link condition (or quality) is an essential component to construct a wireless network topology. It is not uncommon for a node to select a good link for packet delivery. In power-constrained networks, such as Wireless Sensor Networks (WSNs), an even complex task could involve adjusting the transmission power for energy saving.

In previous studies, most of works used metrics like Received Signal Strength Indication (RSSI), Signal-to-Noise Ratio (SNR) and Signal-to-Interference plus Noise-Ratio (SINR) which were calculated at the packet level to reflect the channel conditions. However, such metrics could be misleading [1]. For example, RSSI could only exist in successfully received packets. Much useful intermediate information cannot be obtained. For SNR or SINR, the external interference could introduce packet loss even if the measured SNR is high [2]. This is because the measurement may not be capable to distinguish and minimize transient signal variation during the whole packet transmission.

We argue that packet level metrics are insufficient to indicate the link condition. In particular, they are unable to tell what is happening in the transmission. We propose to analyze more fine-grained information from the lower layer of the network protocol stack. We have conducted this measurement study at the physical layer by taking IEEE 802.15.4 standard as
TABLE I: Symbol to Chip Mapping

\begin{tabular}{|c|c|c|c|}
\hline 4-bit Symbol & 32-chip PN-Code & 4-bit Symbol & 32-chip PN-Code \\
\hline 0x0 & 0x744AC39B & 0x8 & 0xDEE06931 \\
0x1 & 0x44AC39B7 & 0x9 & 0xEE06931D \\
0x2 & 0x4AC39B74 & 0xA & 0xE06931DE \\
0x3 & 0xAC39B744 & 0xB & 0x06931DEE \\
0x4 & 0xC39B744A & 0xC & 0x6931DEE0 \\
0x5 & 0x39B744AC & 0xD & 0x931DEE06 \\
0x6 & 0x9B744AC3 & 0xE & 0x31DEE069 \\
0x7 & 0xB744AC39 & 0xF & 0x1DEE0693 \\
\hline
\end{tabular}

an example. We classify link conditions by chip error pattern analysis.

A chip is an accessible element at the physical layer for technologies, such as IEEE 802.15.4 and 802.11b standards, which employ Direct Sequence Spread Spectrum (DSSS). An information bit is repackaged as some sequences of chips before being transmitted over the air. A chip is a fraction of a packet or even a bit. Since the chip duration is much shorter, we believe that it can capture more information in channel changes due to attenuation, interference and multipath. Hence, analyzing their error patterns could reflect the channel conditions. Such analysis can be performed regardless the checksum of the frame which is an advantage over the packet level metrics.

Early studies have identified many important characteristics of packet delivery. Majority of them made the hypothesis that packet loss are caused by attenuation, multi-path effect [3] or interference. But in most cases, these effects were neither quantified nor empirically measured which leave these hypotheses unevaluated.

In our experiments, we explore the error patterns based on IEEE 802.15.4 standard. We have conducted extensive experiments to discover and verify several notable chip error patterns under attenuation and interference environments. To the best of our knowledge, this is the first detailed, systematic experiment study of chip-level error characteristics in IEEE 802.15.4 standard.

The rest of this paper is organized as follows. In Section II, we give some background information. This is followed by experimental methodology in Section III. The results for thoughtful analysis of chip error patterns are presented in Section IV. Furthermore, a brief overview on relate literature 


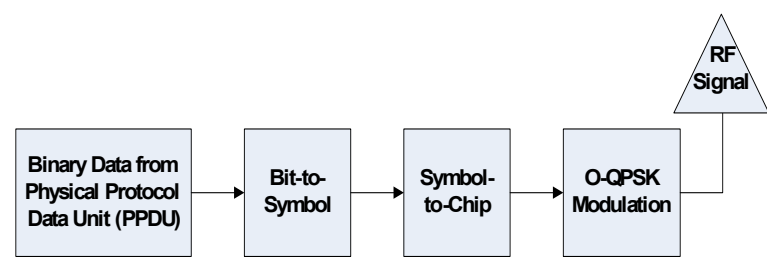

Fig. 1: Block diagram of the $2.4 \mathrm{GHz}$ modulation and spreading of IEEE 802.15.4

is given in Section V. Finally, conclusions are presented and suggestions are made for future research.

\section{BACKGROUND}

In this section, we will give a brief overview of IEEE 802.15.4 standard on which our findings are based. We will focus on the specifications which is relevant to our experiments and measurements. For our study, the most important part is PN-Code in DSSS and CEPP. The later one is the metric we will use in later discussions.

\section{A. PN-Code in DSSS of IEEE 802.15.4}

IEEE 802.15.4 standard specifies PHY and MAC sublayers for low-rate wireless personal area networks (LR-WPANs). It targets at ubiquitous devices for low-cost and low-speed communication. IEEE 802.15.4 standard defines channels in three spectrum bands: $868 \mathrm{MHz}, 915 \mathrm{MHz}$ and $2.4 \mathrm{GHz}$. Among these, the $2.4 \mathrm{GHz}$ channels lie in one of the most popular shared ISM band that various wireless technologies and microwave oven operate. Such a complex environment motivates our study of channel conditions in this paper.

IEEE 802.15.4 networks use DSSS that is also adopted in IEEE $802.11 \mathrm{~b}$. DSSS converts one or more bits of data to a codeword (PN-Code) that is usually longer than the original data. A bit in a PN-Code is called a chip; thus a PN-Code is also known as a chip sequence. In IEEE 802.15.4, a 4-bit data symbol is mapped to a 32-chip PN-Code as shown in Table I.

At the sender side, an $n$-byte frame is encoded to $2 n \mathrm{PN}$ Codes. The PN-Code is modulated to baseband transmission waveform and is ultimately transmitted over the air (Figure 1). At the receiver side, a correlator is responsible for decoding received chip sequences to corresponding information bits. The received chips may contain errors and cannot seek the perfectly matched PN-Codes as in Table I. It accepts them under certain threshold and find a best-match PN-Code for a chip sequence. In the ideal case, the 'best-match' PN-Code should be exactly equal to the captured chip sequence; whereas in real situations, it is not always the case. Although a sender should never transmit a wrong PN-Code (a chip sequence not included in Table I), some chips could be corrupted in the presence of interference. In case of corrupted chips, however, the 'best-match' PN-Code needs not to fully agree with the erroneous chip sequence.

\section{B. Chip Errors per PN-Code}

To find out a 'best-match' PN-Code there are various methods with respect to different considerations. One such method

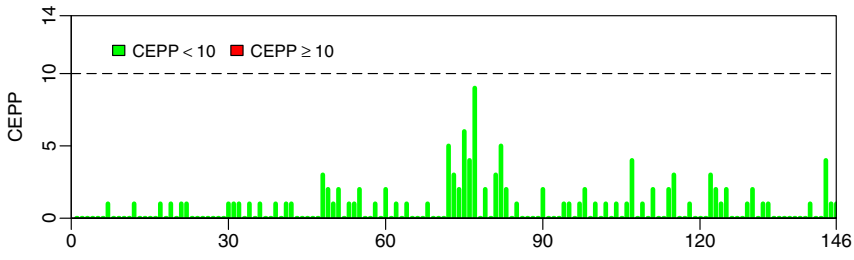

(a) CEPPs in a correctly received frame

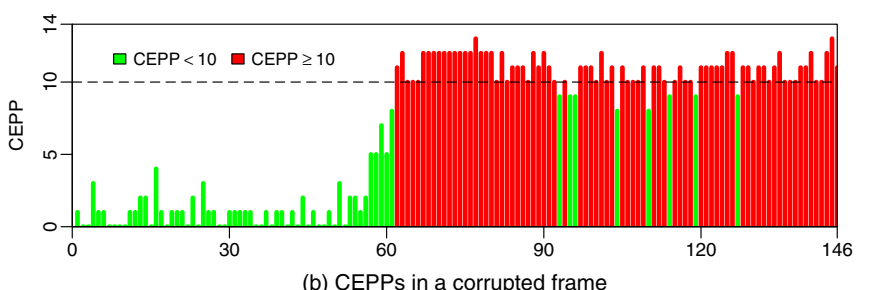

Fig. 2: CEPPs over time $(\boldsymbol{\Lambda})$ for two different frames

is maximum likelihood decoder (MLD) that we choose to conduct our study. In MLD, a 32-bit chip sequence $P$ is compared with 16 predefined PN-Codes $P N_{1}, P N_{2}, \ldots, P N_{16}$ to find out a $k \in\{1,2, \ldots, 16\}$ such that

$$
\underset{k}{\arg \min } h\left(P, P N_{k}\right),
$$

where $h$ is the hamming distance of two bit strings (the number of different positions that the corresponding chips are different).

Given the maximum likelihood match $k$, we then define the value of $h\left(P, P N_{k}\right)$ as Chip Errors per PN-Code (CEPP) which is a key concept in our later discussion. Putting all CEPPs of a frame together, we obtain a time series of CEPPs. It is denoted by $\boldsymbol{\Lambda}$ and the $i$ th CEPP in the time series is denoted by $\boldsymbol{\Lambda}(i)$. Figure 2 plots the CEPPs $(\boldsymbol{\Lambda})$ of two different frames. Each frame consists of an 11-byte header, a 60-byte payload and a 2-byte CRC checksum. Therefore, each frame has 146 CEPPs for 146 received PN-Codes.

If CEPP is less than the correlator error threshold, the data is assumed to be correctly received. In our setup, up to 10 chips out of a total 32 in a PN-Code could be corrupted without negating the receiver's ability to recover from the original data. In Figure 2(a), we can find that all CEPPs are less than 10 which is the threshold. Therefore, this frame can be considered as correctly received. In contrast, if any CEPP in a frame rises beyond the threshold, this frame would be dropped as illustrated in Figure 2(b).

\section{EXPERIMENTAL METHODOLOGY}

\section{A. Transmission Hardware and Software Environment}

Our experiments have taken USRP [4] devices as the transmitter, receiver and interferer. Each of the devices is equipped with the RFX2400 daughterboard for $2.3 \mathrm{GHz}$ and $2.4 \mathrm{GHz}$ communication. The devices are running GNU Radio and 802.15.4 PHY implementation. The original version of 
the 802.15.4 PHY code [5] will drop the frame if any of the received chip sequence has more than 10 bit differences. We have modified it to allow the receiver to continue the decoding process even it encounters a chip sequence with more than 10 bit differences till the end of the frame.

\section{B. Experimental Environment}

In order to verify our results, the experiments are conducted in various environments, i.e., a chamber and an ordinary indoor office.

A chamber provides us with a shielded room which blocks any external RF from entering the room. The use of special pyramidal RF absorbing materials at the ceiling, floor and walls significantly reduce the reflective surface for RF inside the room. It simulates an ideal environment without any interference from external sources other than our own transmission devices.

An ordinary indoor office is located in our academic building where there are many access points providing $802.11 \mathrm{~b} / \mathrm{g}$ service at $2.4 \mathrm{GHz}$ channels. It serves as a general indoor environment where interference takes place as usual.

\section{Experimental Scenario}

We have conducted experiments in three different scenarios, namely attenuation, interference.

1) Attenuation: Attenuation is the reduction of signal strength as it propagates through the space. It is caused by a complex set of factors, including the propagation medium, reflection and propagation distance. The experiments are conducted in our office. In these experiments, we use two USRPs as the transmitter and the receiver. To avoid the interference from $802.11 \mathrm{~b} / \mathrm{g}$ devices which are operating at $2.4 \mathrm{GHz}$ ISM band, we have shifted our communication channels to $2.375 \mathrm{GHz}$. We have verified that there are no users using this channel during our experiments.

First, we set two USRPs in a straight line with a clear path between them. This allows a line-of-sight (LOS) propagation. Before collecting the data, we have calibrated and recorded the set of transmission power which makes the link in the transitional region. In the experiment, we send 2000 frames with length of 73 bytes at $10 \mathrm{~ms}$ interval with random payload content. The experiment is repeated 100 times with different transmission power within the transitional region.

2) Interference: Due to the broadcast nature of the wireless signal, concurrent transmitting links may interfere each other. This is a fundamental issue in wireless communication. To examine the interference effect, we have conducted experiment in the chamber to avoid uncontrollable interferers and multipath effects. In the chamber, there are no interference sources except those we deliberately introduced. We have a sender and receiver pair together with an interference source. Without the interference source. The transmission power of the transmitter has been configured to allow the receiver to receive and decode all frames correctly in the absence of interference source.

An USRP running 802,15.4 code acts as an interferer. It broadcasts at a constant rate of $250 \mathrm{Kbps}$ which performs as a
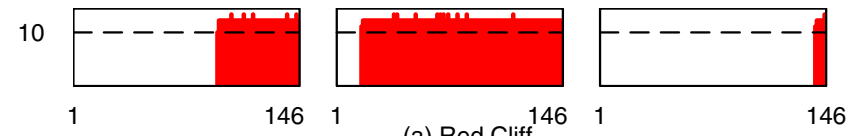

10
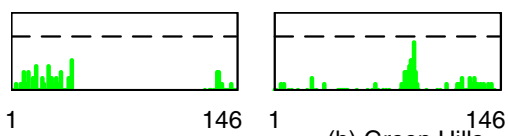

(b) Green Hills

10
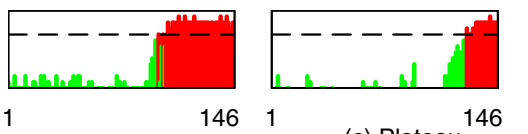

(c) Plateau
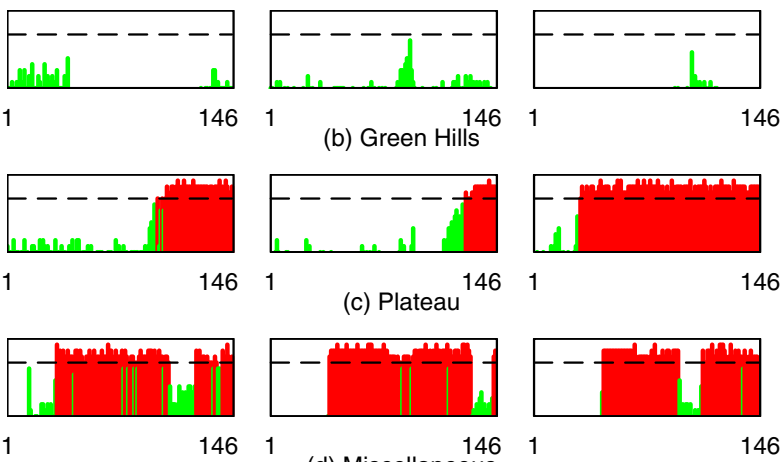

(d) Miscellaneous

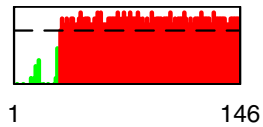

146

Fig. 3: Four major chip error patterns

802.15.4 'hidden terminal' for sender and induces frame loss. The transmission power of interferer varies in the experiments and results in different PRR for investigating effects of strong and weak interferences.

The sender sends 2000 frames at $10 \mathrm{~ms}$ interval. The frame size and time interval of the interference source are changed randomly. The experiment is repeated 100 times for different sets of interferer parameters.

\section{Chip Error Patterns}

During the measurement study on GNU Radio [6] testbed based on IEEE 802.15.4, we have identified several notable distinct patterns at chip-level with respect to CEPP as shown in Figure 3.

Figure 3 depicts several CEPP time. The $\mathrm{x}$-axis is the index of CEPP (from 1 to 146) and the $y$-axis is the value of CEPP. In order to clearly understand these patterns, we color the bars with red and green according to whether the CEPP is beyond the threshold value. A red bar means the corresponding CEPP is greater than or equal to the threshold while a green bar means the contrary. This figure displays four major chip error patterns that we observed in experiments. We name these patterns as (a) Red Cliff, (b) Green Hills, (c) Plateau and (d) Miscellaneous, respectively. We now give a detailed description as well as mathematical definition of each pattern. Note that for the convenience of later discussion, we will define an extra Non-Error pattern which is actually not an 'error' pattern.

1) Non-Error Pattern (NE): If there is no chip error in the received frame, we call this kind of 'error pattern' as NonError pattern or NE in short.

Definition The pattern of a CEPP time series $\boldsymbol{\Lambda}$ is Non-Error (NE) if $\boldsymbol{\Lambda}(i)=0, i \in\{1,2, \ldots,|\boldsymbol{\Lambda}|\}$.

2) Red Cliff Pattern (RC): For Red Cliff pattern, we can see from Figure 3(a) that almost all CEPPs in a frame are shown as red bars. When the first red bar appears, red bars will last 
to the end of the frame. Additionally, green bar should not appear in this pattern.

Definition The pattern of a CEPP time series $\boldsymbol{\Lambda}$ is Red Cliff (RC) if there exists a $k \in\{1,2, \ldots,|\boldsymbol{\Lambda}|-1\}$ such that $\boldsymbol{\Lambda}(i)=0, i \in\{1,2, \ldots, k\}$ and $\boldsymbol{\Lambda}(i)>=10, i \in$ $\{k+1, k+2, \ldots,|\boldsymbol{\Lambda}|\}$.

3) Green Hills Pattern $(G H)$ : There is another pattern that only has green bars. In Figure 3(b) we see that green bars scatter all over the frame.

Definition The pattern of a CEPP time series $\boldsymbol{\Lambda}$ is Green Hills $(\mathrm{GH})$ if it is NOT a Non-Error pattern and $\boldsymbol{\Lambda}(i)<10, i \in$ $\{1,2, \ldots,|\boldsymbol{\Lambda}|\}$.

4) Plateau Pattern (PL): The characteristic of Plateau pattern is that green bars come before red bars and once a red bar appears it will show up in a row till the end. We can also take Plateau pattern as a Green Hills pattern followed by a special Red Cliff pattern.

Definition The pattern of a CEPP time series $\boldsymbol{\Lambda}$ is Plateau (PL) if it is NOT a Red Cliff pattern and there exists a $k \in$ $\{1,2, \ldots,|\boldsymbol{\Lambda}|-1\}$ such that $0<\Lambda(i)<10$, for $i<k$ and $\boldsymbol{\Lambda}(i)>=10$, for $i>k$.

5) Miscellaneous Pattern (MC): We introduce Miscellaneous pattern to include all CEPP time series that does not belong to any of the above four patterns. This pattern may be further divided into several classes for future analysis. In this paper, however, we will not go into details of Miscellaneous patterns.

Definition The pattern of a CEPP time series $\Lambda$ is Miscellaneous (MC) if it is NOT a Non-Error, Red Cliff, Green Hills or Plateau pattern.

The above five patterns are the major patterns shown in our experiments. In the next section, we will systematically analyze these patterns in various environments through extensive experiments. It is worth pointing out that we will use the abbreviation and full name of a pattern interchangeably from here on.

\section{EXPERIMENTAL Result ANALysis}

In this section, we will describe our experiments and analysis in detail. We have conducted experiments in two different scenarios, namely attenuation and interference. We must reiterate that the chips under study are over-the-air chips, and not the information bits. We believe the chip-level information can give us more details because of a shorter duration for a chip than a packet. Some studies have revealed the existence of three reception regions based on packet reception rate and SNR for wireless links: connected, transitional, and disconnected [7].

\section{A. Metrics}

In the first place, we define four metrics with respect to patterns as well as the Packet Reception Rate (PRR) that will be used later. We use the terminology $N(A)$ indicating the number of CEPP time series that is of pattern $A$.

$$
\begin{gathered}
P R R=\frac{N(N E)+N(G H)}{\text { Number of transmitted frames }} \\
\alpha_{G H}=\frac{N(G H)}{N(G H)+N(N E)} \times 100 \% \\
\beta_{R C}=\frac{N(R C)}{N(R C)+N(P L)+N(M C)} \times 100 \% \\
\beta_{P L}=\frac{N(P L)}{N(R C)+N(P L)+N(M C)} \times 100 \% \\
\beta_{M C}=\frac{N(M C)}{N(R C)+N(P L)+N(M C)} \times 100 \%
\end{gathered}
$$

In the metric definitions above, $\alpha_{G H}$ is the percentage of frames with GH pattern among all correctly received frames and $\beta_{R C}$ is the percentage of frames with RC pattern among all corrupted frames. $\beta_{G H}$ and $\beta_{M C}$ are the corresponding percentages for PL and MC patterns.

\section{B. Analysis}

In transitional region, the instantaneous link condition is undetermined. The frame may or may not be received. This transitional region is a focus in this study. To the best of our knowledge, other studies on the link condition address only the dropped frames. In this study, we will also present the result and analyze all CEPPs even PRR is extremely high. We will explain why such analysis may be useful in some application scenarios.

Figure 4 plots the distribution of different chip error patterns of 2000 frames for each power level. The result shows that the Red Cliff Pattern is the dominant error pattern in this scenario and always contributes more than $80 \%$ of the total errors regardless of any PRR. The contribution from Plateau and Green Hill patterns are negligible.

It is not always possible to have LOS transmission. We have also investigated the error patterns for non-line-of-sight (NLOS) scenarios. We put a metallic cabinet between the transmitter and the receiver. The PRR will decrease sharply but similar error patterns are observed. We have excluded the figures for brevity.

Figure 5 plots the distribution of different chip error patterns of 2000 frames for every set of interferer parameters. Plateau and Green Hill patterns are the dominant patterns. The sum of these two patterns contribute around $80 \%$ of errors for most values of the PRR. Unlike the results from attenuations, the Red Cliff patterns in 802.15.4 interference is close to zero. Such characteristics hints that the distribution of different chip error patterns may be used to distinguish the link condition between attenuation and 802.15.4 interference. 


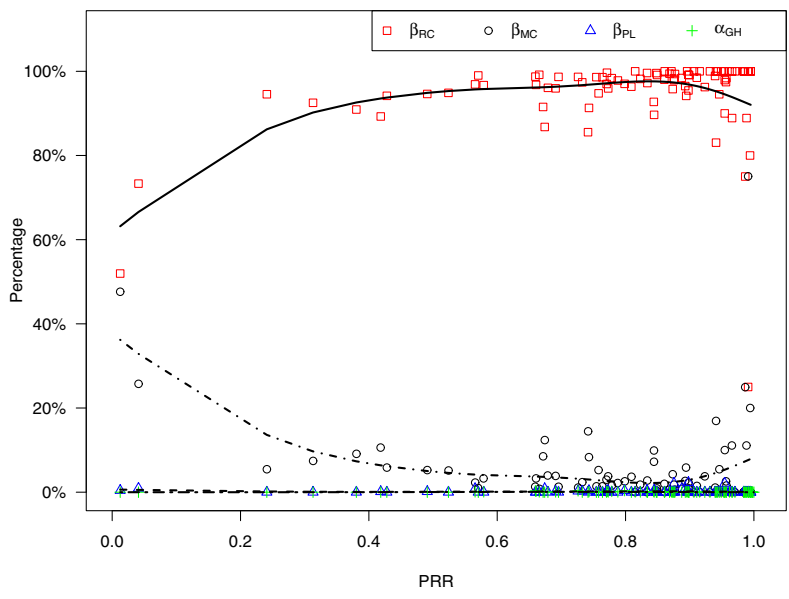

Fig. 4: Chip error patterns in the attenuation scenario

\section{RELATED WORK}

Some researchers reported measurement results of bit and packet errors study in IEEE 802.11 [8] [9]. Aguayo et al. [10] analyzed the packet loss in Roofnet network and stated that link errors stayed relatively uniform in static wireless networks. Han et al. [11] identified three bit error patterns and verified their presence with different physical environments and hardware. They claimed these errors were caused by hardware, and not induced by channel fading. Jamieson et al. [12] use PHY-independent hints which is based on Hamming distance in block decoding to develop a partial packet recovery system. Rayanchu et al. [13] presented a study based on bit error patterns of received data for loss diagnosis based on OFDM in 802.11. Their scheme, called COLLIE, computed the metrics on the single data packet that was received in error. However, in our experiments, we found out that not only the error packets will give us information, but also the correctly received ones. And COLLIE required the receiver to send back the whole corrupted packets to the sender, which introduces a great overhead.

\section{CONCLUSIONS AND FUTURE WORK}

In this paper, we conducted extensive experiments to study the chip error patterns in IEEE 802.15.4. We observed that there are four major error patterns, namely Red Cliff, Green Hills, Plateau and Miscellaneous. We also showed that different channel conditions would result in various distributions of error patterns. Further analysis on these patterns may help us to characterize and infer channel condition.

In previous sections, we assume that the link is invariant within a specified period. It may not be true for all the scenarios, such as environments with moving people. The time-variant nature of the link could prevent our algorithm to predict the future link condition. These scenarios are left as the future work to be explored.

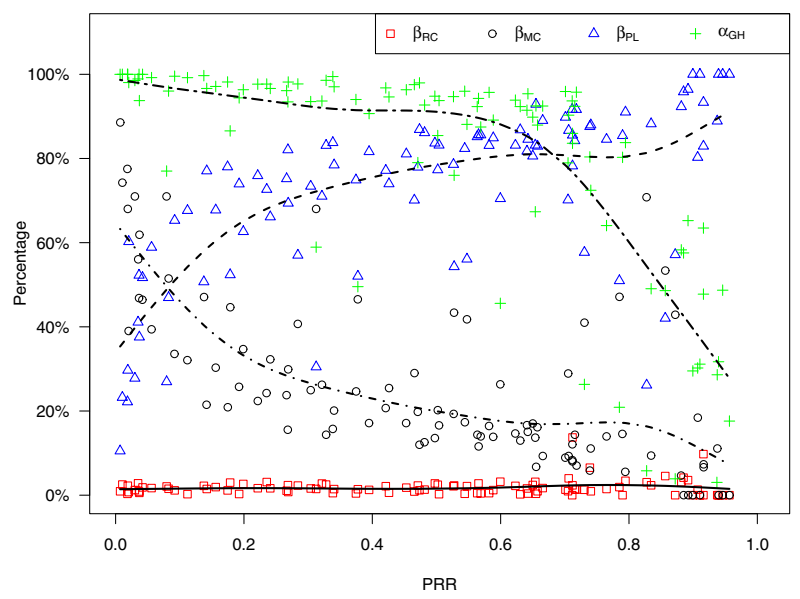

Fig. 5: Chip error patterns with interference from IEEE 802.15.4 source

\section{ACKNOWLEDGMENTS}

This research was supported in part by Hong Kong RGC Grant HKUST617908, China NSFC Grant 60933011, the National Basic Research Program of China (973 Program) under Grant No. 2006CB303000, the National Hi-Tech R\&D Program of China (863 Program), the National Science and Technology Major Project of China under Grant No. 2009ZX03006-001, and the Science and Technology Planning Project of Guangdong Province, China under Grant No. 2009A080207002.

\section{REFERENCES}

[1] C. Reis, R. Mahajan, M. Rodrig, D. Wetherall, and J. Zahorjan, "Measurement-based models of delivery and interference in static wireless networks," in Proceedings of ACM SIGCOMM, 2006.

[2] J. Zhang, K. Tan, J. Zhao, H. Wu, and Y. Zhang, "A practical snr-guided rate adaptation," in proceedings of IEEE INFOCOM, 2008.

[3] M. Zuniga and B. Krishnamachari, "Analyzing the transitional region in low power wireless links," in proceedings of IEEE SECON, 2004.

[4] M. Ettus, "The Universal Software Radio Peripheral or USRP, 2008."

[5] T. Schmid, "GNU Radio 802.15. 4 En-and Decoding," UCLA NESL Technical Report, Tech. Rep., 2005.

[6] E. Blossom, "Gnu software defined radio," http://www.gnu.org/software/ gnuradio.

[7] J. Zhao and R. Govindan, "Understanding packet delivery performance in dense wireless sensor networks," in proceedings of ACM Sensys, 2003.

[8] D. Eckhardt and P. Steenkiste, "Measurement and analysis of the error characteristics of an in-building wireless network," Proceedings of ACM SIGCOMM, 1996.

[9] A. Willig, M. Kubisch, C. Hoene, A. Wolisz et al., "Measurements of a wireless link in an industrial environment using an IEEE 802. 11compliant physical layer," IEEE Transactions on Industrial Electronics, vol. 49, no. 6, pp. 1265-1282, 2002.

[10] D. Aguayo, J. Bicket, S. Biswas, G. Judd, and R. Morris, "Link-level measurements from an $802.11 \mathrm{~b}$ mesh network," in Proceedings of ACM SIGCOMM, 2004

[11] B. Han, L. Ji, S. Lee, B. Bhattacharjee, and R. R. Miller, "All bits are not equal a study of ieee 802.11 communication bit errors," in Proceedings of IEEE INFOCOM, 2009.

[12] K. Jamieson, "PPR: Partial packet recovery for wireless networks," in Proceedings of ACM SIGCOMM, 2007.

[13] S. Rayanchu, A. Mishra, D. Agrawal, S. Saha, and S. Banerjee, "Diagnosing wireless packet losses in 802.11: Separating collision from weak signal," in Proceedings of IEEE INFOCOM, 2008. 